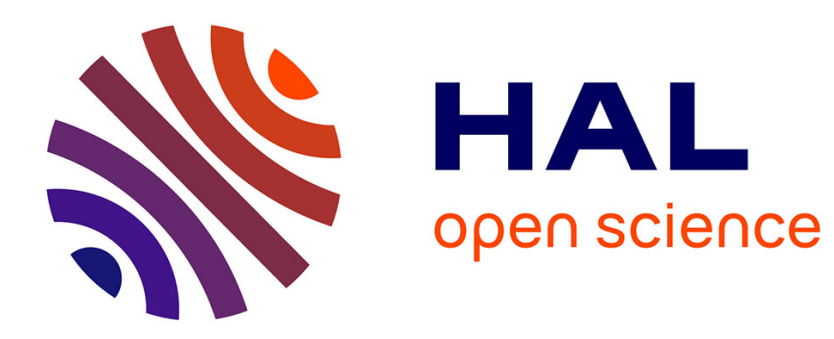

\title{
Le Brésil, géant de l'Amérique latine
}

Gérard-François Dumont

\section{To cite this version:}

Gérard-François Dumont. Le Brésil, géant de l'Amérique latine. Population et avenir, 2005, 674, pp.4-7, 20. 10.3917/popav.674.0004 . halshs-00770359

\section{HAL Id: halshs-00770359 \\ https://shs.hal.science/halshs-00770359}

Submitted on 22 Jun 2013

HAL is a multi-disciplinary open access archive for the deposit and dissemination of scientific research documents, whether they are published or not. The documents may come from teaching and research institutions in France or abroad, or from public or private research centers.
L'archive ouverte pluridisciplinaire HAL, est destinée au dépôt et à la diffusion de documents scientifiques de niveau recherche, publiés ou non, émanant des établissements d'enseignement et de recherche français ou étrangers, des laboratoires publics ou privés. 


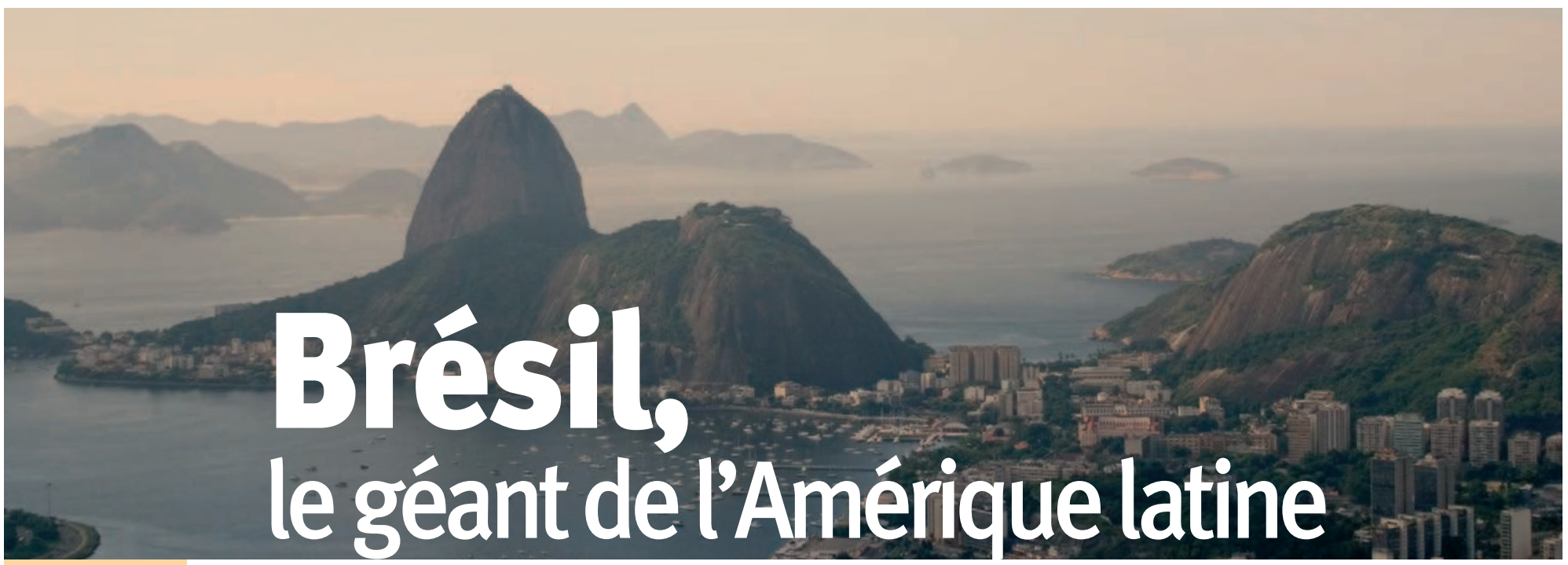

Avec une population estimée à 184 millions en 2005, le Brésil se classe numéro deux du continent américain derrière les États-Unis

(296 millions) et cinquième État le plus peuplé du monde ${ }^{1}$. Il représente $\mathbf{2 0 , 7} \%$ de la population de l'Amérique, $33 \%$ de la population de l'Amérique latine et $49 \%$ de celle de l'Amérique du Sud. Lors du premier recensement effectué au Brésil en 1872, ce pays ne comptait que 10 millions d'habitants, ce qui signifie que sa population a été multipliée par 18 en 130 ans. Comment expliquer une telle croissance ? Et un tel rythme va-t-il se poursuivre ? par Gérard-François DUMONT

\section{Une croissance stimulée} par l'immigration.e.

n fait, il est bien connu que la cr oissance démographique du Brésil a été longtemps, au X IX siècle et au premier quart du XX ${ }^{\mathrm{e}}$ siècle, soutenue par une très impor tante immigration européenne qui atteignit son apogée de 1900 à la guerr e de 1914-1918. Auparavant, les flux migratoires avaient été plus modestes, même si les estimations de la période pré-statistique ont une précision limitée. Il est néanmoins possible d'indiquer qu'au $X V l^{e}$ siècle, le peuplement européen concerne presque exclusivement les terres brésiliennes du Nordeste, avec deux centres principaux d'attraction : Recife Olinda et Salvador de Bahia. L'extension du peuplement à l'intérieur des terres ne devient significativ e qu'au XVII ${ }^{\mathrm{e}}$ siècle, avec l'exploitation de la canne à sucr e, qui accélère la traite des Noirs d'Afrique, et l'installation de fermes d'élevage.

$\mathrm{Au} X \mathrm{XVIII}$ e siècle, une nouvelle extension géographique du peuplement s'effectue à l'intérieur des terr es, avec l'extraction de l'or, qui explique la croissance démographique du Minas Ger ais, puis du Goiás et du Mato Gr osso. Les colons portugais affluent tandis que la tr aite négrière se poursuit. En raison des évolutions de la géogr aphie du peuplement, la capitale est transférée en 1770 de Bahia à Rio de Janeiro. Mais, malgré les appor ts migratoires, les effectifs de population sont encor e très faibles, compte tenu de l'importante superficie du territoire : 8557 milliers de $\mathrm{km}^{2}$ (seize fois la France métropolitaine). Ils sont
1. LES ANNÉES DE FORTE IMMIGRATION AU BRÉSIL

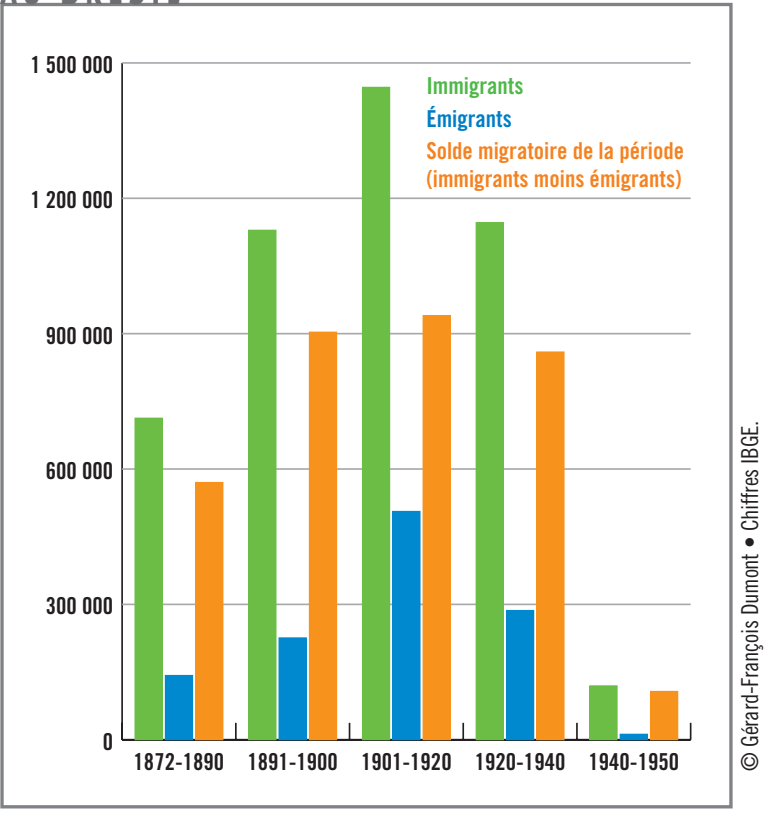

estimés à 2,4 millions en 1808, 4,4 millions en 1819, 4,9 millions en $1823,7,7$ millions en 1854 et enfin 10,1 millions lors du premier recensement de 1872.

Puis, l'immigration fournit un apport d'autant plus important que l'on encourage la venue d'immigrants, en raison des besoins en main-d'œuvr e d'une économie qui se fonde alors sur la culture du café.

Pour l'ensemble de la période allant de 1872 à 1950, l'immigration totalise 4,5 millions de personnes mais, compte tenu des estimations de l'émigration, le solde migratoire total est estimé à 3,38 millions de personnes, dont la présence exerce bien évidemment des effets sur l'accr oissement naturel. Celui-ci, pendant la seconde moitié du XX e siècle, devient la cause quasi ex clusive de la cr oissance démographique brésilienne, alors que le pays parcourt sa période de transition démographique ${ }^{2}$.

\section{... puis démultipliée par la transition démographique}

Jusque dans les années 1930, les taux annuels de natalité et de mortalité du Brésil $r$ estent très élevés, $r$ espectivement aux alentours de $46 \%$ et de $24 \%$. En conséquence, le taux d'accroissement naturel (différence entre le taux de natalité et le taux de mor talité) est élevé : environ $22 \%$. 


\section{LE MOUVEMENT NATUREL AU BRÉSIL}

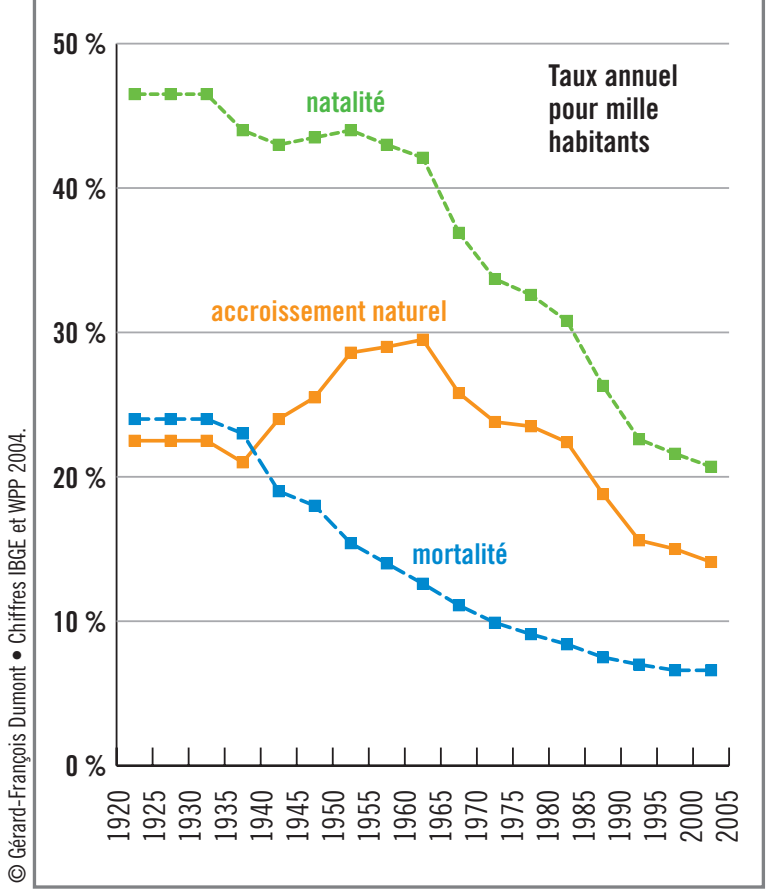

Additionnant les effets de ce taux et les apports migratoires, la population du Brésil augmente : 18,2 millions d'habitants au recensement de 1900, 27,5 millions en 1920, avant d'atteindre 41,2 millions en 1940. Puis, les évolutions démographiques commencent à pr endre en compte les effets de la première étape de la transition démographique qui débute dans les années 1940. L e déclenchement de la baisse des mor talités infantile, infanto-adolescente et

\section{LA FÉCONDITÉ ET LA MORTALITÉ INFANTLE AU BRÉSIL}

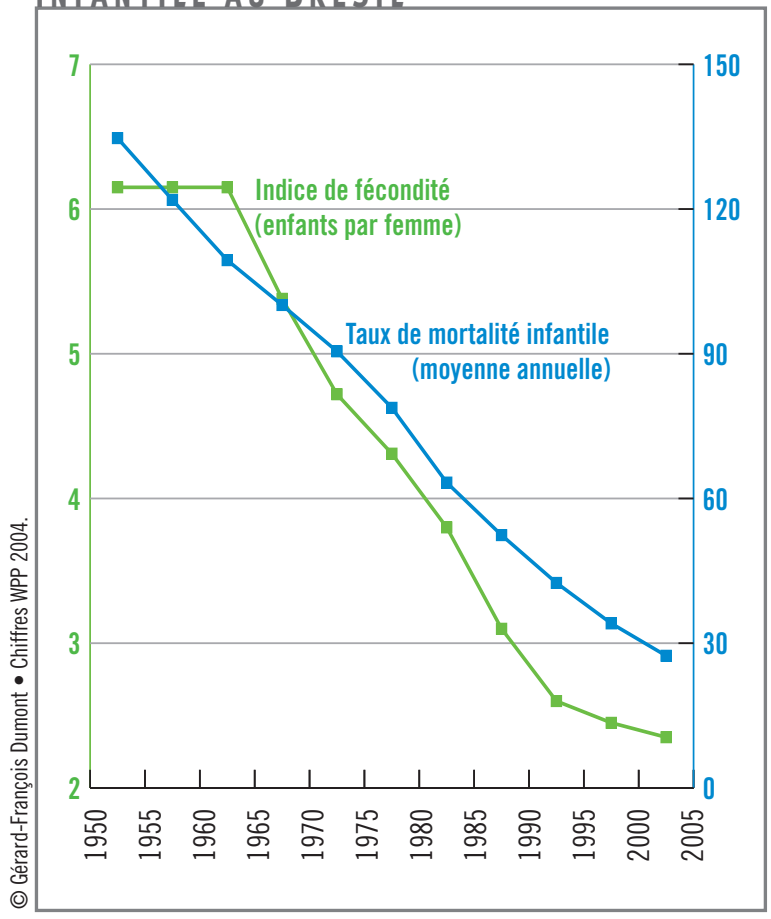

maternelle, engendre une diminution du taux de mor talité. Comme, à la même période, le taux de natalité demeur e dans une four chette élevée, entr e 42 et $44 \%$ o jusqu 'en 1965, le taux d'accroissement naturel passe de $22 \%$ à la fin des années 1930 à un maximum de 29,5\% \% dans la seconde moitié des années 1960. La cr oissance démographique brésilienne s'intensifie donc des années 1940 aux années 1960, portant la population à 53,9 millions en 1950, 72,7 millions en 1960 et 95,9 millions en 1970.

Puis, les progrès permettant de fair e reculer la mortalité se prolongent. Par exemple, le taux de mortalité infantile baisse de 134 décès d'enfants de moins d'un an pour mille naissances au début des années 1950 à 27 un demi-siècle plus tard. Autrement dit, si, en 1950, plus de tr eize nouveaux-nés sur cent décédaient av ant d'atteindre l'âge d'un an, il n'y en a plus que 2,7 en moyenne en 2005. Une telle diminution de $80 \%$ contribue à une réduction des deux tiers du taux de mortalité entre les années 1930 et la fin des années 1990.

À compter des années 1960, la population réalisant que les taux de mor talité sont durablement abaissés, la fécondité s'adapte au nouveau régime de la mor talité permis par les formidables progrès hygiéniques et sanitair es. En conséquence, la fécondité entame une baisse de plus de 6 enfants par femme au début des années 1960 à 2,4 envir on vers 2005 , soit une division par presque trois en quarante ans.

\section{Les deux retournements démographiques}

Le suivi des évolutions permet de dater les deux retournements démographiques du Brésil. L e premier se situe à mi-parcours de la tr ansition démographique, lorsque la baisse du taux de natalité devient plus rapide que celle du

\section{LES DEUX RETOURNEMENTS DÉMOGRAPHIOUES AU BRÉSIL}

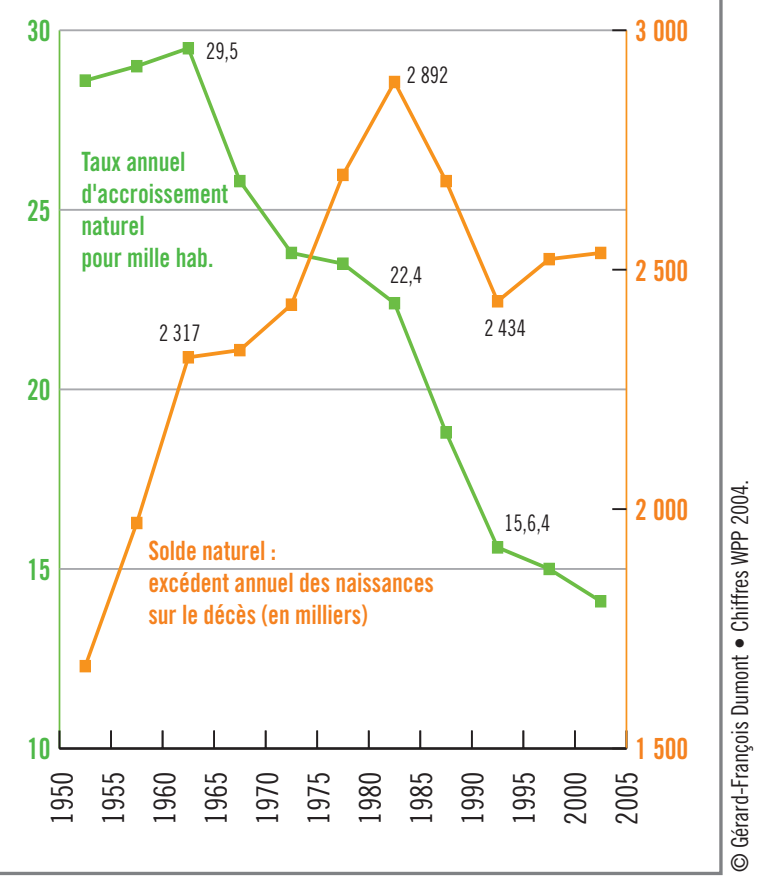


taux de mortalité. Le taux d'accroissement naturel entame en conséquence, au milieu des années 1960, une diminution : ce premier retournement démographique installe le Brésil dans la seconde étape de la tr ansition démographique. Le taux d'accroissement naturel baisse de moitié en quarante ans, de 29,5\% vers 1965 à 14,1 \%o au début des années 2000.

Jusqu'aux années 1980, en dépit de la diminution du taux d'accroissement naturel, l'excédent annuel des naissances sur les décès continue de cr oître, car les génér ations en âge de fécondité sont très nombr euses. Puis, dans les années 1980, le niveau de fécondité, qui poursuit sa diminution, finit par entraîner un second retournement à la baisse, celui du solde naturel, de l'excédent des naissances sur les décès. Ce solde natur el, après avoir passé par un maximum de 2,9 millions au début des années 1980, se réduit aux envir ons de 2,5 millions au début des années 2000.

\section{Un début de vieillissement}

En conséquence, les effectifs des génér ations les plus jeunes deviennent moindres que ceux des générations précédentes. La pyramide des âges de 2005 met bien en évidence ce phénomène, avec des générations de jeunes enfants (0-4 ans) moins nombreuses que les enfants de 5 9 ans, de 10-14 ans ou encore de 15-19 ans. Ainsi le Brésil de 2005 voit-il diminuer ses effectifs de jeunes à scolariser dans les écoles primaires. La proportion des moins de 15 ans, qui était de plus de $43 \%$ de la population en 1965 , baisse régulièrement et n'est plus que de $27,9 \%$ en 2005. Et la proportion des personnes âgées de 60 ans ou plus augmente progressivement. La pyramide des âges prend donc une forme de sapin, puisque la population commence à vieillir.

Quelle va être l'intensité de ce vieillissement?

Elle dépend notamment de la fécondité et de son évolution. Or, les données les plus fines indiquent en 2005 une fécondité brésilienne en dessous du seuil de $r$ emplacement des génér ations. En effet, compte tenu en par ticulier d'une

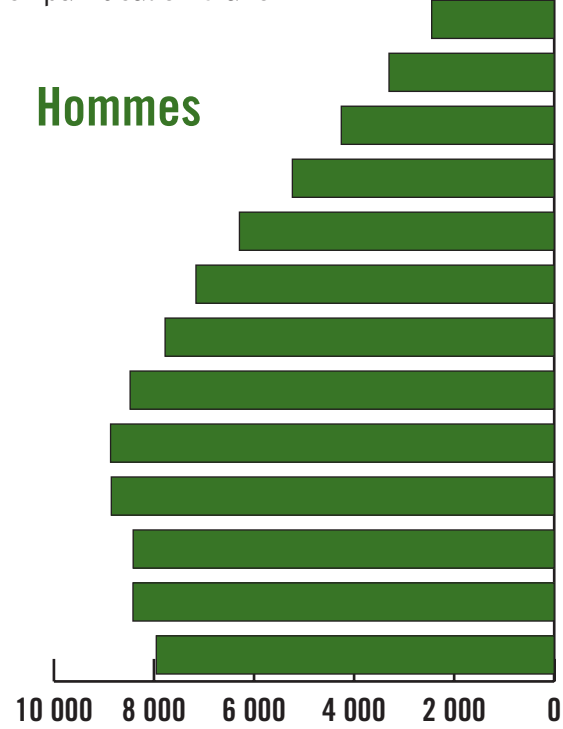

mortalité infantile cer tes abaissée, mais encor e cinq fois supérieure à celle de la France, le seuil de simple remplacement au Brésil est de 2,35 enfants par femme (et non de 2,1 comme en France). Or, la fécondité, selon le Census bureau, est à 1,93 enfant par femme ${ }^{3}$, donc nettement inférieur e à ce seuil. Comment expliquer une telle situation ? Il faut, pour la comprendre, ajouter les spécificités brésiliennes en matière de contrôle de la fécondité et d'accouchement.

Dans ce pays, en effet, la stérilisation s'est imposée comme une forme de contraception, et l'âge à la stérilisation tend à baisser : il concerner ait le cinquième des femmes ayant moins de 25 ans. En outre, la « césarienne » est considérée comme une forme « moderne " d'accouchement, d'où une fréquence de césariennes parmi les plus élevées au monde, atteignant le tiers des naissances, des césariennes qui, à leur tour, débouchent, après la deuxième ou tr oisième naissance, sur une ligatur e des trompes, donc sur une stérilisation.

Il résulte de ce qui précède que l'av enir démographique du Brésil n'est en rien celui qui av ait été projeté et qui tablait sur plus de 200 millions d'habitants dès 2000 . Selon l'hypothèse moyenne, les effets de vitesse acquise porteraient la population du Brésil à 253 millions en 2050 , donc à un rythme considérablement ralenti par rapport à la seconde moitié du XXe siècle. Mais, comme la décélération démographique du Brésil est rapide, la Division de la population des Nations Unies présente une hypothèse basse selon laquelle la population de ce pays pourr ait diminuer à la fin des années 2030 , av ec des rythmes très différenciés selon les régions.

\section{D'importants changements géographiques}

En 1872, le Brésil a une densité mo yenne de 1,2 habitant $/ \mathrm{km}^{2}$. Compte tenu de la croissance démographique, l'augmentation de la densité peut par aître spectaculaire, puisque qu'elle est multipliée par dix-sept en 130 ans, mais ne conduit toutefois qu'à une densité de 21 habitants $/ \mathrm{km}^{2}$ en 2005.

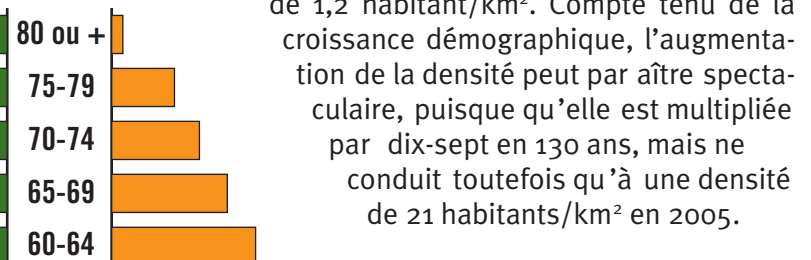

$60-6$

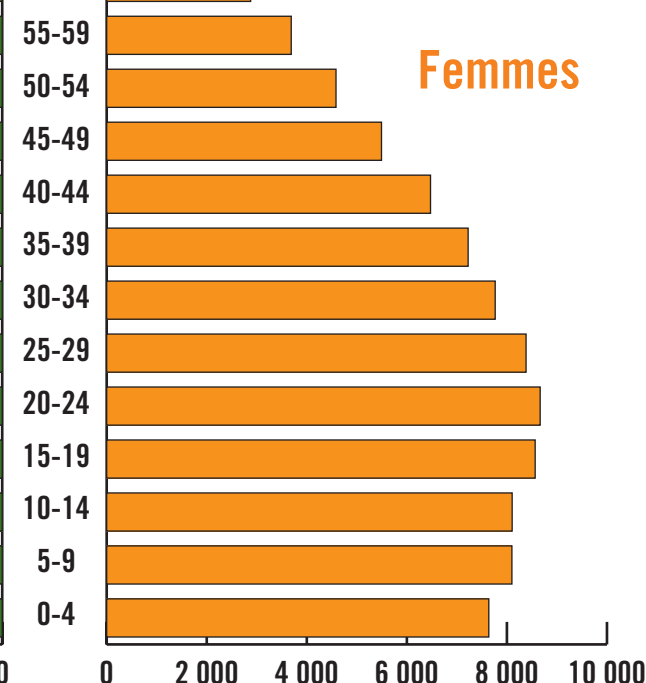




\section{Les processus de migrations internes}

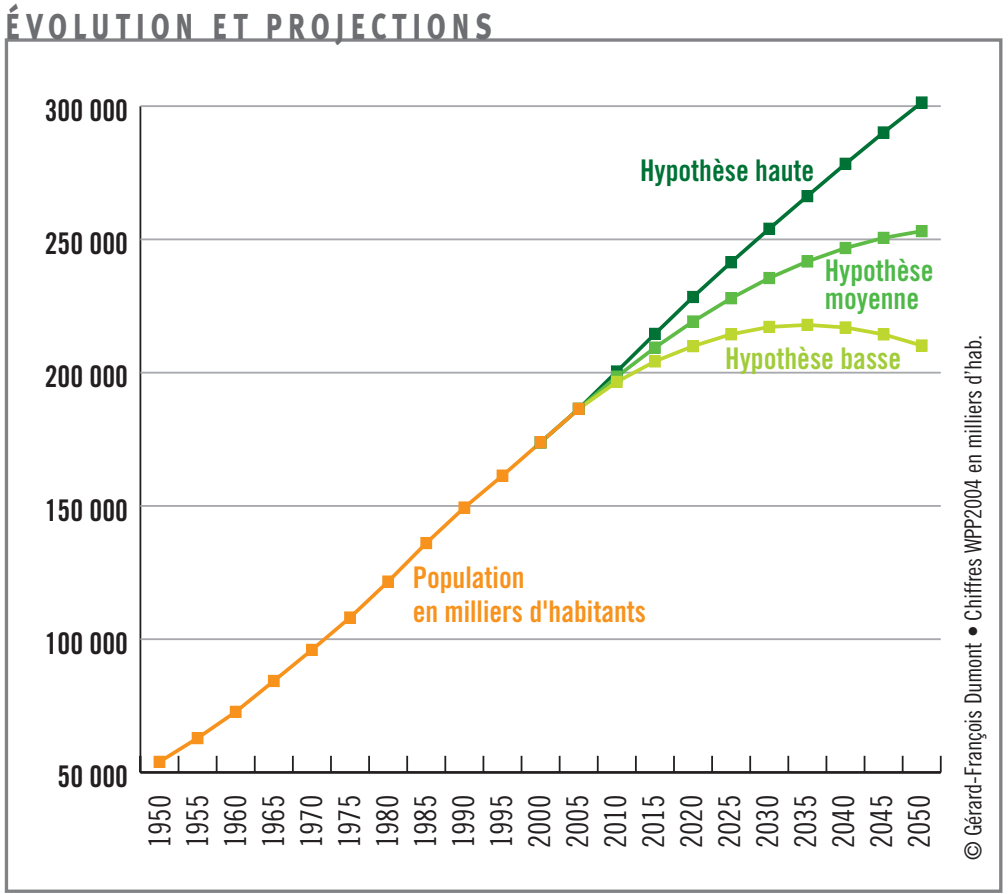

Les changements dans la répartition géographique du peuplement sont à relier avec les évolutions des structures économiques et les différences de dynamisme économique. $L$ es régions du Nordeste ou du Sudeste (Minas Gerais) ont été historiquement les moteurs de l'économie coloniale brésilienne, ayant bénéficié de quasi-rentes économiques lors des cycles du sucre, de l'or ou de l'élev age. Elles sont désormais génér alement des régions d'émigr ation, essentiellement interne, notamment vers la mégapole de São Paulo qui est le grand pôle économique du Brésil. Car, dans la riv alité entre les deux mégapoles, Rio de Janeiro et São Paulo, cette dernière l'a nettement emporté ces dernières décennies. Rio (agglomération de 11,2 millions d'habitants en 2003) a per du son rôle de capitale et subi le déménagement de services ministériels et administratifs,

Cette évolution de la densité ne s'est pas faite de façon géographiquement homogène sur le territoir e brésilien, qui comprend 26 États ${ }^{4}$ plus le district fédéral de Brasilia, et que l'on divise en cinq grandes régions : le Nordeste, le Sudeste, le Sud, le Centre-Ouest et le Nord qui comprend l'essentiel de l'Amazonie.

Au premier recensement de 1872 , la région la plus peuplée est celle du Nordeste, qui représente alors 46,7\% de la population totale. Au début des années 2000 , le poids démographique relatif de ce Nor deste, qui compte neuf États, n'est plus que de $28 \%$.

En revanche, le Sudeste compte désormais $42,6 \%$ de la population du Brésil, dont l'État le plus peuplé, celui de São Paulo avec 38 millions d'habitants. Le centre de gravité démographique du Brésil s'est donc déplacé du nordest vers le sud et plus précisément vers le centre-sud. Car le Centre-Ouest - où le Brésil a fondé la capitale fédér ale Brasilia en 1960 - qui ne comptait que 2,2 \% de la population du pays en 1872 , en regroupe désormais $6,8 \%$. De même, le Nord, qui regroupe sept des États de l'Amazonie, a vu son poids démogr aphique augmenter, même s'il demeure faible, passant des alentours de $4 \%$ au début du $X X^{e}$ siècle à 7,6 \% au début du XXI ${ }^{\mathrm{e}}$ siècle.

Ainsi, l'occupation du territoire brésilien se poursuit, mais les densités de population sont encor e très faibles dans de nombreux États ${ }^{5}$. La région Nord, c'est-à-dire l'Amazonie, dépasse à peine 3 habitants $/ \mathrm{km}^{2}$, le Centre-Ouest a vu sa densité multipliée par 54 depuis 1872, mais cela ne représente, au début des an nées 2000 , que 7 habitants $/ \mathrm{km}^{2}$. Le Nordeste, qui avait 3 habitants $/ \mathrm{km}^{2}$ au premier recensement de 1872, en compte 31 au début des années 2000. Des cinq grandes régions, la plus dense est le Sudeste, av ec 79 habitants $/ \mathrm{km}^{2}$, ce qui est encor e moins que la France métropolitaine et trois fois moins que le Royaume-Uni. Quant à la densité de la région Sud, elle est désormais de 44 habitants $/ \mathrm{km}^{2}$.

tandis que son secteur industriel est nettement plus réduit que celui de São P aulo (agglomération de 17,9 millions d'habitants en 2003), l'État de São Paulo étant le plus riche des 26.

Un autre type de flux migratoires internes tient à la volonté du gouvernement, symbolisée par la création de la capitale fédérale en 1960, d'aménager le territoir e en dév eloppant des infrastructures dans les États les moins denses ou en ouvr ant de nouveaux fronts pionniers agricoles, tentative partielle de réponse à la question foncièr e, jamais véritablement résolue. Ainsi, parmi les États de " l'Amazonie légale », c'est-à-dire ceux faisant partie de la politique régionale concernant l'Amazonie, les plus for tes croissances démographiques de la dernière décennie s'expliquent par l'immigr ation interne : elles concernent le Para, l'État le plus peuplé de la région Nord, qui compte l'agglomération de Belém, 2 millions d'habitants en 2003, le Maranhão, État situé au nor d-ouest du Nordeste et appartenant à ce que le Brésil appelle l’Amaz onie légale.

Si l'on considère exclusivement la population rurale, elle est souvent en décroissance, mais elle a nettement augmenté, dans la dernière décennie, dans l'État d'Amaz onas, encore considéré comme un fr ont pionnier. Mais génér alement, l'émigration rurale, phénomène qui por te le nom « d'invasion rurale " au Brésil, compte tenu de son intensité, se poursuit, avec pour effet une urbanisation ${ }^{6}$ souvent désordonnée. Il en résulte un taux d'urbanisation ${ }^{7}$ de $81 \%$, particulièrement élevé pour un pays dont le développement exige de nouveaux progrès.

1. Ce qui explique son souhait de présence au Conseil de sécurité de l'ONU, Cf. Dumont, Gérard-François, « A I'ONU, le Sud veut compter » Population \& Avenis, $n^{\circ} 665$, novembre-décembre 2003

2. Période, de durée et d'intensité variables, pendant laquelle une population passe d'u régime démographique de mortalité et de natalité élevées à un régime de basse mortalité, puis de faible natalité $\mathrm{Cf}$ Dumont, Gérard-Francois, Les populations du monde, Paris, Éditions Armand Colin deuxième édition, 2004

3 . Même si ce chiffre était considéré comme sous-évalué, et que l'on prenne la moyenne entre ce chiffre du Census burea et celui du Population reference bureau, 2,5 enfants par femme certainement surévalué, le résultat donnerait une fécondité inférieure au seuil de remplacement des générations, donc un taux net de reproduction inférieur à l'unité

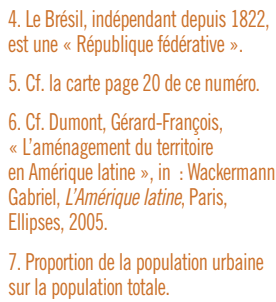




\section{La densité et l'armature ubaine du Brésil}

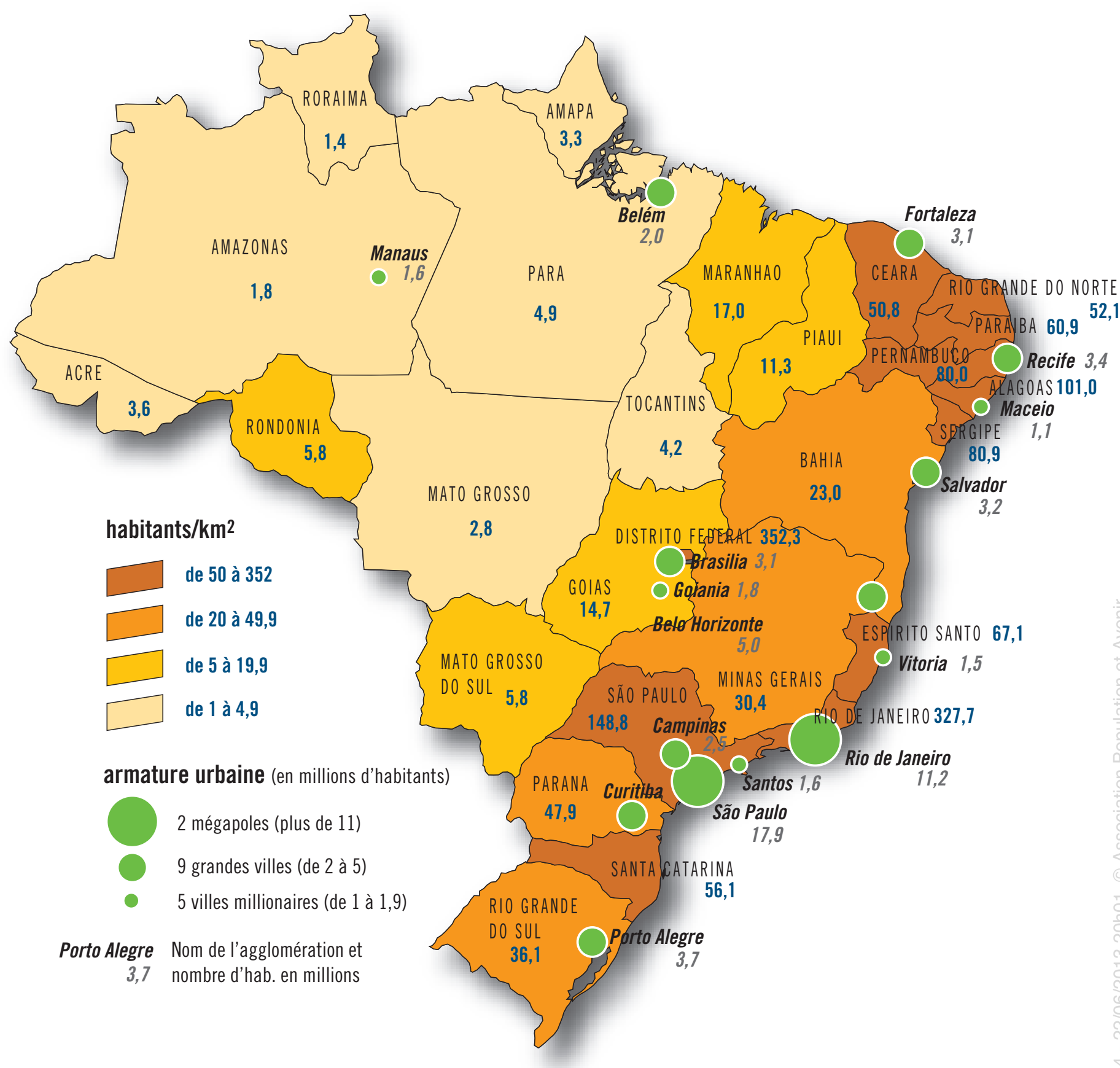

Armature urbaine : Outre les deux grandes mégapoles de Sao Paulo (17,9 millions d'habitants) et de Rio de Janeiro (11,2 millions), le Brésil compte 9 agglomérations multimillionnaires, dont la plus peuplée est Belo Horizonte ( 5 millions d'habitants) et 5 villes millionnaires.

Superficie : Le Brésil, avec $8557 \mathrm{~km}^{2}$, compte une superficie seize fois supérieure à celle de la France métropolitaine, représentant $46 \%$ de celle de l'Amérique du Sud et $42 \%$ de l'Amérique latine.

\section{Population \& Avenir}

9, rue du Faubourg-Poissonnière, 75009 Paris
Population : Avec 179 millions d'habitants en 2005, le Brésil compte la première population d'Amérique latine, soit 32,6\% de la population latino-américaine et $40 \%$ de la population sud-américaine.

Densité : Avec 21 habitants $/ \mathrm{km}^{2}$ en 2005, le Brésil est peu dense. La population du Brésil est très inégalement répartie selon les 26 Etats de la « république fédérative s». Les densités les plus élevées se constatent dans les territoires des premières colonies de peuplement, donc dans les Etats maritimes du Nordeste et dans certains du Sud-Est, auxquels s'ajoute le district fédéral de Brasilia. Les plus faibles densités se constatent dans les Etats de l'Amazonie. 